\title{
Raised plasma endothelin in unstable angina and non- $Q$ wave myocardial infarction: relation to cardiovascular outcome
}

Cardiovascular Research Unit, University of Edinburgh, Edinburgh I Wieczorek K A A Fox University Department of Medicine, Western General Hospital, Edinburgh W G Haynes D J Webb

Department of Haematology, Royal Infirmary of Edinburgh, Edinburgh C A Ludlam

Correspondence to: Professor Keith A A Fox, Cardiovascular Research Unit, Hugh Robson Building, George Square, Edinburgh EH8 9XF.

Accepted for publication 14 June 1994 Iwona Wieczorek, William G Haynes, David J Webb, Christopher A Ludlam,
Keith A A Fox

\begin{abstract}
Background-Among patients with independent evidence of coronary disease and recent onset unstable angina or non-Q wave myocardial infarction the incidence of subsequent cardiovascular events is high. Markers predictive of adverse cardiac outcome in unstable angina and non- $Q$ wave myocardial infarction need to be defined more accurately. Endothelin-1 is a potent endothelium derived vasoconstrictor peptide that may play a part in the pathophysiology of acute myocardial ischaemia.

Aim and study design-In a study that specifically identified high risk patients a group of 16 consecutive patients with either unstable angina at rest or non- $Q$ wave myocardial infarction were prospectively investigated to establish whether these conditions are associated with high plasma immunoreactive endothelin and whether endothelin concentration at presentation is related to cardiovascular events within the next 12 weeks. Controls consisted of a group of 40 healthy subjects.
\end{abstract}

Results-Patients had significantly higher mean (SD) plasma endothelin at presentation than did healthy controls $(7.4(1.1)$ v 5.0 (1.2) pg/ml, $P<0.0001)$. At nine weeks plasma endothelin was still significantly higher in those patients who had subsequent cardiovascular events, $(n=9$, acute myocardial infarction or refractory angina with electrocardiographic changes and revascularisation procedures, 8.5 (2.6) $\mathrm{pg} / \mathrm{ml}, \mathrm{P}<0.005 v$ controls) whereas its concentration returned to normal in those patients who had a favourable outcome ( $n=7$, $5.9(0 \cdot 7) \mathrm{pg} / \mathrm{ml})$. Compared with those patients who had an uneventful course, patients with subsequent events had significantly higher plasma endothelin, both at presentation and at nine weeks ( $P<0.05$ on both occasions).

Implications-Endothelin may contribute to the pathophysiology of acute coronary syndromes and may relate to subsequent cardiovascular outcome.

(Br Heart f 1994;72:436-441)

Patients presenting with recent onset unstable angina or non- $Q$ wave myocardial infarction constitute a group that is at high risk of pro- gression to further myocardial infarction or sudden death. ${ }^{1}$ These patients have not been shown to benefit from thrombolysis, ${ }^{2}$ and a proportion of them fail to stabilise on antianginal treatment and aspirin and may experience recurrent episodes of myocardial ischaemia prompting revascularisation. ${ }^{1}$ In the antithrombotic therapy in acute coronary syndromes (ATACS) study there was no difference in the cardiovascular outcome at three months after presentation between the group with unstable angina and that with non$Q$ wave infarction. ${ }^{3}$ The search continues for reliable markers to facilitate early identification of those patients with acute coronary syndromes who are at a high risk of developing subsequent cardiovascular events, in whom early intervention may be indicated.

Intracoronary thrombosis as a result of a disrupted atherosclerotic plaque is regarded as the pivotal mechanism in acute coronary syndromes and the angiographic and pathological findings are not dissimilar in unstable angina and non- $Q$ wave infarction. ${ }^{4-6}$ Clinically, the two diagnoses may not be distinguishable at presentation and both are associated with the release of sensitive markers of cardiac muscle injury, such as troponin T. ${ }^{7}$ Abnormalities of haemostasis ${ }^{8-10}$ and increased coronary reactivity to constrictor stimuli ${ }^{11}$ have been suggested as possible pathogenetic factors in acute coronary syndromes. Previous studies have shown the importance of activation of platelets and of the coagulation system in the pathophysiological mechanism of acute myocardial ischaemia, ${ }^{8-10}$ whereas the role of endogenous vasoconstrictor substances has so far received less attention.

Endothelin is a potent endothelium derived vasoconstrictor peptide in animals ${ }^{12}$ and humans. ${ }^{13}$ Raised plasma concentrations of endothelin have recently been reported in patients with acute myocardial infarction and in a heterogeneous group of patients with symptomatic atherosclerosis. ${ }^{14} 15$ In these the plasma endothelin concentration was positively correlated with the number of sites of atherosclerotic involvement but not with age. Patients with stable angina have, on the other hand, been shown to have normal plasma endothelin concentrations. ${ }^{14} 16$

Our aim was to investigate prospectively whether unstable angina and non- $Q$ wave myocardial infarction are associated with changes in plasma immunoreactive endothelin and whether raised endothelin concentrations are associated with indicators of adverse cardiovascular prognosis. 


\section{Patients and methods}

\section{PATIENT SELECTION}

This study was performed on patients recruited for a multicentre trial of ATACS, which compared the effectiveness of combination antithrombotic treatment with aspirin and anticoagulation $v$ aspirin alone in patients with unstable angina and non- $Q$ wave myocardial infarction. ${ }^{1}$ The inclusion criteria were $(a)$ age $>21$; $(b)$ ischaemic chest pain due to unstable angina or non- $Q$ wave myocardial infarction, with the qualifying episode of chest pain within the 48 hours before recruitment to ATACS, and (c) additional evidence of ischaemic heart disease was required. This evidence consisted of at least one of the following: (a) electrocardiographic changes during pain on admission suggesting ischaemia-for example, ST segment depression or elevation, or $\mathrm{T}$ wave inversion; (b) previous myocardial infarction; $(c)$ history of typical exertional angina, with chest pain precipitated by effort and relieved by rest or glyceryl trinitrate, or a previously positive exercise test; (d) previous coronary angiography showing $\geqslant 50 \%$ luminal narrowing in any coronary artery. Patients with $Q$ wave infarction at presentation were excluded from ATACS. As stipulated by the protocol, no patient was treated with thrombolysis, and coronary angiography was performed on clinical indications only.

Based on screening forms, 4.5 patients were screened for each patient identified for the study. Among 51 patients recruited into the ATACS study in our centre, prospective endothelin measurements were undertaken in the last 16 consecutive patients. Our control group consisted of 40 healthy volunteers, mean (SD) age 34 (17), range 20-78. Eleven control subjects were in the same age range as the patients (59 (13), range 40-74).

\section{STUDY DESIGN}

The patients were prospectively followed up for a period of 12 weeks with assessments at least every three weeks in the cardiology clinic. Blood samples were taken on the first morning after admission and at nine weeks of follow up from all patients, except for two patients from whom follow up samples could not be obtained for technical reasons. The controls were sampled on one occasion only. Venous blood was taken from an antecubital vein into chilled potassium EDTA tubes, put immediately on ice and centrifuged at $4000 \mathrm{rpm}$ for $30 \mathrm{~min}$ at $4^{\circ} \mathrm{C}$. Plasma was stored at $-70^{\circ} \mathrm{C}$ until assay. Clinicians were blinded to the results of endothelin measurements.

\section{ANALYTICAL PROCEDURES}

Plasma immunoreactive endothelin was measured by radioimmunoassay. SepPak C18 silica columns (Waters Associates, Milford, MA, USA) were equilibrated by washing with methanol $(5 \mathrm{ml})$, distilled water $(5 \mathrm{ml})$, and then $4 \%$ acetic acid $(5 \mathrm{ml})$. Each $2 \mathrm{ml}$ plasma sample was diluted with $3 \mathrm{ml}$ of $4 \%$ acetic acid and loaded on to a column; these were washed with $25 \%$ ethanol $(3 \mathrm{ml}$ ), and eluted with $4 \%$ acetic acid in $86 \%$ ethanol $(2 \times 1 \mathrm{ml})$. The eluates were evaporated under nitrogen in a water bath at $37^{\circ} \mathrm{C}$, and reconstituted in borate buffer of $\mathrm{pH} 8.4$ $(2 \mathrm{ml})$. Duplicate extracted samples and standards containing 1 to $48 \mathrm{pg} / \mathrm{ml}$ of endothelin-1 (each $200 \mu \mathrm{l}$ ) were incubated with rabbit polyclonal antibody raised against endothelin-1 (ITS Production BV, Wijchen, The Netherlands; in $100 \mu \mathrm{l}$ distilled water) and ${ }^{125}$ I-endothelin-1 (ITS; in $100 \mu$ distilled water). After vortexing, tubes were incubated for $18 \mathrm{~h}$ at $4^{\circ} \mathrm{C}$. Donkey antirabbit $\gamma$ globulin bound on solid phase (ITS; $100 \mu \mathrm{l}$ ) was added to all tubes, except those for total counts, and the tubes were incubated for 30 min at room temperature. The amount of radioactivity in the antibody bound fraction was determined by $\gamma$ counting for $2 \mathrm{~min}$. The recovery of added endothelin-1 was $84 \%$. Within and between assay coefficients of variation were $2 \cdot 4 \%(n=6)$ and $4 \cdot 2 \%(n=5)$. The sensitivity of this assay was $1 \mathrm{pg} / \mathrm{ml}$ endothelin. Cross reactivity of the assay with endothelin-1, endothelin-2, endothelin-3, and proendothelin-1 was $100 \%, 52 \%, 96 \%$, and $7 \%$.

\section{STATISTICAL ANALYSIS}

Initial statistical analyses were performed on the whole group of patients with unstable angina and non- $Q$ wave infarction (primary analysis, $n=16$ ). Subsequent more detailed comparisons were carried out separately for the two diagnostic categories (subsidiary analysis: unstable angina, $\mathrm{n}=10$, and non- $\mathrm{Q}$ wave myocardial infarction, $n=6$ ).

Statistical analyses were performed with Minitab statistical software (Minitab, Release 7). All values are expressed as mean (SD). Individual comparisons between groups were carried out with unpaired $t$ test. Categorical data were analysed by $\chi^{2}$ test, with Yates' correction, when appropriate. Correlation coefficients wère calculated with Pearson's test. Two tailed tests were performed and $P$ values $<0.05$ were considered to be significant.

\section{Results}

\section{STUDY PATIENTS}

The table shows the main characteristics of the 16 patients. None was hypertensive at the time of the study. Coronary artery disease was verified in all patients by at least one of the criteria: electrocardiographic changes on admission (ST segment depression or elevation or $\mathrm{T}$ wave inversion, $\mathrm{n}=14(88 \%)$ ), cardiac catheterisation performed during the 12 weeks of follow up or before admission $(n=8(50 \%))$ and a history of myocardial infarction $(n=7(44 \%))$. At presentation all patients were in Killip class $I$, and at nine weeks all patients but one were free of left ventricular failure.

UNSTABLE ANGINA $V$ NON-Q WAVE INFARCTION Although all 16 patients fulfilled criteria for unstable angina on admission (all class IIIB 
Demographic and epidemiological data

\begin{tabular}{lccc}
\hline & All & Unstable angina & Non- $Q$ wave MI \\
\hline No & 16 & 10 & 6 \\
Age (yr): & & & $62(10)$ \\
$\quad$ Mean (SD) & $62(8)$ & $62(12)$ \\
Range & $44-77$ & $50-76$ & $4-78$ \\
Sex ratio (F/M) & $4 / 12$ & $3 / 7$ & $1 / 5$ \\
Duration of angina (yr): & $5 \cdot 1(7 \cdot 5)$ & $7 \cdot 3(8 \cdot 7)$ & $1 \cdot 4(2 \cdot 1)$ \\
Mean (SD) & 2 & 4 & $0 \cdot 3$ \\
Median & $0-25$ & $0-25$ & $0-5$ \\
Range & $7(44 \%)$ & 6 & 1 \\
Previous MI & $2(13 \%)$ & 1 & 1 \\
Previous CABG/PTCA & $8(50 \%)$ & 7 & 1 \\
Coronary angiography: & $3(38 \%)$ & 2 & 1 \\
Total & $1(12 \%)$ & 1 & 0 \\
1 vessel & $4(50 \%)$ & 4 & 0 \\
2 vessel & $14(88 \%)$ & 9 & 5 \\
3 vessel & $10(63 \%)$ & 9 & 1 \\
Ischaemic electrocardiogram at presentation & $16(100 \%)$ & 10 & 6 \\
Medical treatment: & $14(88 \%)$ & 8 & 6 \\
Heparin & $12(72 \%)$ & 10 & 2 \\
Aspirin & $13(81 \%)$ & 9 & 4 \\
Beta-blockers & $347(631)$ & $58(26)$ & $901(812)$ \\
Calcium antagonists & $28-2339$ & $28-101$ & $335-2339$ \\
Nitrates & &
\end{tabular}

CABG, coronary artery bypass grafting; MI, myocardial infarction; PTCA, percutaneous transluminal coronary angioplasty. according to Braunwald, ${ }^{17}$-for example, chest pain at rest within preceding $48 \mathrm{~h}$ ), six patients were subsequently shown to have enzymatic evidence of an evolving non-Q wave myocardial infarction (increase in cardiac enzymes, no electrocardiographic evidence of new $Q$ waves). Patients with unstable angina did not differ significantly from those with non- $Q$ wave infarction with respect to age, sex distribution, smoking habits, history of hypertension and acute myocardial infarction, incidence of ischaemic electrocardiogram at presentation, and treatment with aspirin, $\beta$-blockers, calcium chan-

Figure 1 Plasma endothelin concentrations were significantly higher in patients with unstable angina or non- $Q$ wave myocardial infarction $(n=16)$, both at presentation and at nine weeks than in the controls

$(n=40)$
$\star \star \star P$

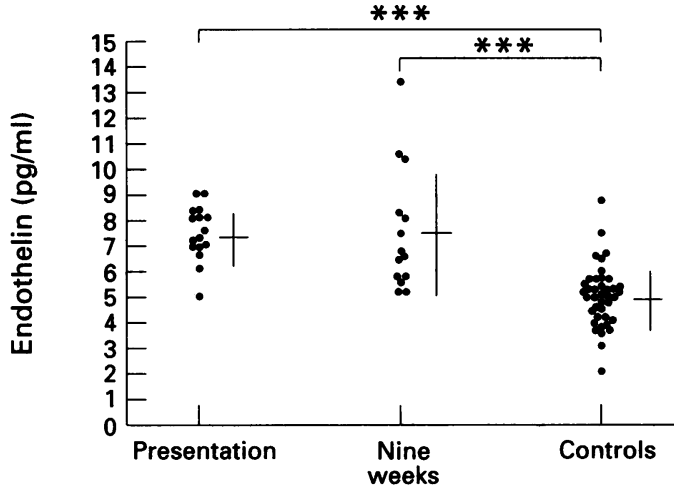

Figure 2 Cardiovascular outcome analysis. Plasma endothelin concentrations were significantly higher in patients with subsequent cardiac events $(n=9)$ than in those who remained event free $(n=7)$, both at presentation and at nine weeks. $* P<0.05$; $\star * P<0.005$ $\star \star \star P<0.0001$.

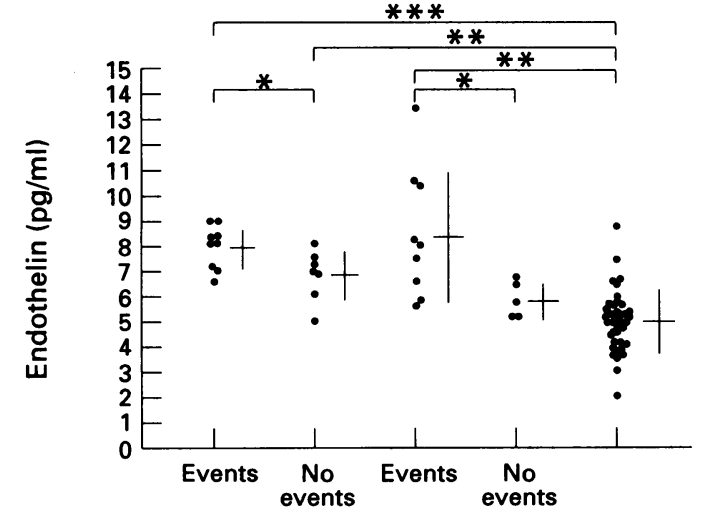

Presentation Nine weeks Controls nel blockers, and nitrates (table). They tended to have a longer history of previous angina and a higher rate of performed angiography, but these trends did not reach significance. Significantly more patients with unstable angina $(n=9)$ were treated with heparin or anticoagulation regimens on admission and during follow up, compared with patients with non- $\mathrm{Q}$ wave myocardial infarction $(\mathrm{n}=1$, $\left.\chi^{2}=8.6, P<0.005\right)$.

\section{CARDIOVASCULAR OUTCOME}

In the primary analysis of all 16 patients with acute coronary syndromes, nine (56\%) patients had cardiovascular events during 12 weeks of follow up, whereas seven patients remained event free or had only mild angina. The cardiac events were as follows: four patients had episodes of refractory angina with electrocardiographic changes despite maximal medical treatment, one developed acute $Q$ wave myocardial infarction, and four underwent surgical revascularisation on account of evidence of continuing ischaemia (three coronary artery bypass grafting (CABG) and one percutaneous transluminal coronary angioplasty (PTCA)). In none of the cases did a myocardial infarction or a revascularisation take place within four weeks of the nine week sample being taken. Of the four patients with episodes of refractory pain one patient refused angiography, two had inoperable coronary artery disease, and in one the clinical symptoms resolved and he did not proceed to angiography during the study period.

Subsidiary analysis showed that the subsequent event rate among patients with unstable angina was six of 10 (three CABG, one PTCA, two angina with electrocardiographic changes), whereas three of six patients with subendocardial infarction had subsequen events (one myocardial infarction, two angina with electrocardiographic changes). The event rate at 12 weeks did not differ between unstable angina and non-Q wave infarction groups $\left(\chi^{2}=0 \cdot 15, P=N S\right)$.

\section{ENDOTHELIN CONCENTRATIONS}

In the primary analysis of all 16 patients (fig 1), plasma endothelin was significantly raised both at presentation $(7 \cdot 4(1 \cdot 1) \mathrm{pg} / \mathrm{ml})$ and at nine weeks $(7.6(2.4) \mathrm{pg} / \mathrm{ml})$, compared with healthy controls $(5.0(1.2) \mathrm{pg} / \mathrm{ml}$, $P<0.0001$ on both occasions). At presentation, those patients who developed subsequent events $(8.0(0.9) \mathrm{pg} / \mathrm{ml})$ and those who remained event free $(6.9(1.0) \mathrm{pg} / \mathrm{ml})$ had higher endothelin concentrations than the controls $(P<0.0001$ and $P<0.005$; fig 2$)$. At nine weeks, when compared with the controls, endothelin remained significantly raised in those patients who had an unfavourable progress $(8.5(2.6) \mathrm{pg} / \mathrm{ml}, \mathrm{P}<0.005 v$ controls), but it returned to normal values in those patients who remained asymptomatic $(5.9(0.7) \mathrm{pg} / \mathrm{ml})$. Patients with subsequent events had significantly higher endothelin than those who remained event free, both at presentation $(P<0.05)$ and at nine weeks 
( $P<0.05$, fig 2). One of the patients had signs and symptoms of mild left ventricular failure nine weeks after presentation, but the exclusion of this patient did not affect the results from the nine week analysis.

In the analysis based on diagnosis patients with unstable angina $(7.9(0.9) \mathrm{pg} / \mathrm{ml})$ and those with non- $Q$ wave myocardial infarction $(6.8(1 \cdot 1) \mathrm{pg} / \mathrm{ml})$ had significantly higher endothelin concentrations at presentation than healthy controls $(P<0.0001$ and $P<0.01)$. There was a small but significant difference in endothelin concentrations at presentation between patients who presented with unstable angina and those who presented with a subendocardial infarction $(7.9(0.9) v$ $6.8(1.1) \mathrm{pg} / \mathrm{ml}, \mathrm{P}<0.05)$. At nine weeks, endothelin remained higher both in the unstable angina $(7 \cdot 8(2 \cdot 5) \mathrm{pg} / \mathrm{ml})$ and the non-Q wave infarction groups $(7 \cdot 3(2 \cdot 5) \mathrm{pg} / \mathrm{ml})$ than in the control population $(P=0.0002$ and $P<0.005)$. At nine weeks there was no difference in the endothelin concentrations between patients who initially presented with either unstable angina or subendocardial infarction.

All comparisons between patients and controls were subsequently repeated with the age matched control group $(n=11)$. None of the conclusions were altered nor were the measures of significance abrogated by this analysis.

In view of the highly significant $P$ values obtained for the above comparisons, the differences remained significant even after adjustments for multiple comparisons. Comparison of patients with and without events was performed only once at each of the analysed time points and in this instance the results were of borderline significance $(\mathbf{P}=0.042$ at presentation and $\mathbf{P}=0.018$ at nine weeks).

\section{CORRELATION WITH CLINICAI \\ CHARACTERISTICS}

There was no difference in endothelin concentrations on admission between smokers, non-smokers, and ex-smokers $(7 \cdot 3(1 \cdot 1) v 7 \cdot 8$ $(1 \cdot 0) \mathrm{pg} / \mathrm{ml})$ or between patients who received heparin and those who did not $(7 \cdot 8(0.9) v$ $7 \cdot 0(1 \cdot 2) \mathrm{pg} / \mathrm{ml})$. Endothelin concentrations did not differ between men and women $(7 \cdot 3$ $(1 \cdot 1) v 8 \cdot 1(0 \cdot 8) \mathrm{pg} / \mathrm{ml})$. There was no correlation between endothelin concentrations and age, either in the patients $(r=0.23$, $\mathrm{P}=0.38)$ or in the controls $(r=0.06$, $P=0.72)$. We showed no correlation between endothelin concentrations and either systolic $(r=0.38, P=0.14)$ or diastolic blood pressure $(r=0.07, \mathbf{P}=0.8)$ or the number of involved coronary arteries, as found by coronary angiography $(r=0.03$, $P=0.94$ ).

\section{Discussion}

This is the first prospective investigation of the relation between plasma endothelin and cardiovascular outcome in acute coronary syndromes. We have shown a persistent increase of plasma immunoreactive endothelin, lasting for at least nine weeks after admission, in a group of high risk patients presenting with unstable angina or non- $Q$ wave myocardial infarction. Also, we have shown an association between endothelin concentrations at presentation and subsequent cardiovascular outcome.

Previous studies of endothelin in acute myocardial infarction have reported an early rise in plasma endothelin, with a subsequent progressive fall to normal values, completed within 3-14 days of the infarct. ${ }^{14}$ 18-20 Our results, which show that unstable angina and non- $Q$ wave myocardial infarction are associated with high circulating endothelin at presentation, are consistent with those previous findings. Persistently increased circulating endothelin, present only in those patients who developed subsequent cardiovascular events, has not previously been reported.

There have been few previous studies of endothelin concentrations in unstable angina and these have provided conflicting results. ${ }^{162122}$ Ray et al and Stewart et al found no difference in endothelin concentrations in plasma and coronary sinus samples between patients with unstable angina, stable angina, and controls. ${ }^{21} 22 \mathrm{Also}$, in the study by Ray et al endothelin concentrations in unstable angina, although comparable with those in stable angina, were significantly lower than in a group of patients with acute myocardial infarction. ${ }^{21}$ In contrast, in a study of 29 patients with unstable angina, Qiu et al reported that endothelin concentrations were high at presentation compared with healthy controls, and decreased progressively to normal at six hours. ${ }^{16}$ In their study, the size of the initial increase of endothelin concentration in unstable angina was comparable with that found in 29 patients with acute myocardial infarction.

None of the previous studies of endothelin in acute coronary syndromes has provided cardiac outcome data. In our high risk population ( $56 \%$ event rate at 12 weeks) we have shown that endothelin concentrations at presentation may relate to subsequent cardiac events. The event rate in our population was higher than that usually described for unstable angina. It is conceivable that the populations studied by Ray et al and Stewart et al were of relatively lower risk and this may explain why they had no evidence of increased endothelins at the time of presentation. It is not clear, however, why in our study patients with unstable angina tended to have higher endothelin concentration at presentation than the non-Q wave infarction group. Qiu et al also reported a different time course of the increase in endothelins in unstable angina and myocardial infarction: in unstable angina the concentrations decreased progressively to normal values by six hours, whereas in myocardial infarction there was a further increase at $6 \mathrm{~h}$, then a decrease at 12 hours. In the absence of multiple endothelin measurements, we cannot speculate as to whether different dynamics of the endothelin rise 
could have contributed to the reported differences in endothelin concentrations in our patients. It has also to be considered whether these differences could have been related to more extensive use of heparin in our patients with unstable angina than in those who presented with subendocardial infarction. Although the evidence of an effect of heparin on the generation of endothelins is limited, heparin induced inhibition rather than stimulation of endothelin-1 expression and production by cultured endothelial cells has been reported. ${ }^{23}$ In view of these findings, the pattern of heparin use would, if anything, tend to diminish the observed differences in endothelin concentrations between patients with unstable angina and non- $Q$ wave infarction in our population.

In agreement with previous studies ${ }^{1516}$ we did not show a correlation between endothelin concentrations and common clinical characteristics, including age, systolic and diastolic blood pressure, smoking, and the extent of coronary artery disease.

We cannot differentiate which specific endothelin isoforms contribute to the rise in total plasma immunoreactivity in the studied population, because our assay cross reacts substantially with all three isoforms. As endothelin-2 is usually undetectable in plasma $^{23}$ this isoform is unlikely to constitute a large proportion of total immunoreactivity. Thus endothelin-1 and/or endothelin-3 may have contributed to the rise in circulating endothelin. Proendothelin-1 also cross reacts with our antibody, although only to a minor degree $(7 \%)$, and is unlikely to have accounted for the rise in endothelin immunoreactivity.

Both increased generation and decreased clearance of endothelin could have caused the rise in plasma concentrations in patients with coronary syndromes. Plasma creatinine remained normal in all these patients, however, and thus increased generation seems more likely to have accounted for the rise. There is evidence from experimental studies that endothelin production is enhanced by shear stress, impaired release of nitric oxide, and physiological stress. ${ }^{24-26}$ Both thrombin and transforming growth factor- $\beta$ are also known to stimulate endothelin production in cell culture. ${ }^{2728}$ As unstable angina is linked with markers of a hypercoagulable state, ${ }^{8-10}$ it is conceivable that excessive generation of thrombin or release of transforming growth factor- $\beta$ from activated platelets may contribute to increased endothelin concentrations in acute coronary syndromes. This hypothesis would be indirectly supported by previous reports that endothelin concentrations were positively correlated with both thrombinantithrombin III complex and $\beta$ thromboglobulin concentrations in patients presenting with acute myocardial infarction. ${ }^{14}$

Any increase in generation of endothelins in our patients may have been a localised phenomenon or it may have occurred as a systemic process. If the increase occurred in the heart, high local concentrations of endothelin may have been reached. When present in sufficiently high concentrations endothelin may cause direct local or systemic vasoconstriction and potentiate platelet aggregation. ${ }^{29} 30$ In cases of a chronic increase, endothelin may stimulate proliferation of vascular smooth muscle cells in atherosclerotic plaques. ${ }^{31}$ Ultimately, endothelin could be implicated in the pathophysiological mechanism of acute coronary syndromes and its biological actions may actively contribute to myocardial ischaemia. Indeed, there is evidence that antibodies to endothelin limit the infarct size in animal models. ${ }^{32}$ Alternatively, high endothelin concentrations may be a consequence of intermittent episodes of myocardial ischaemia. In either case endothelin could prove to be a useful prognostic marker for adverse outcome in acute coronary syndromes. If confirmed in larger prospective studies, the findings of this study raise a possibility of influencing the cardiovascular outcome in acute coronary syndromes with use of the recently described specific inhibitors to the generation of endothelin. ${ }^{23}$

We gratefully acknowledge the invaluable assistance of Mrs Catriona Simpson.

1 Cohen M, Parry G, Xiong J, Adams PC, Chamberlain D, Wieczorek I, et al, and the ATACS Research Group. Combination antithrombotic therapy in rest unstable Combination antithrombotic therapy in rest unstable angina and non-Q wave infarction. Primary end

2 van den Brand M, van Zijl A, Geuskens R, de Feyter PJ Serruys PW, Simoons ML. Tissue plasminogen activator in refractory unstable angina. A randomized doubleblind placebo-controlled trial in patients with refractory unstable angina and subsequent angioplasty. Eur Heart 1991;12:1208-14.

3 Cohen M, Xiong J, Parry G, Adams P, Chamberlain D, Wieczorek I, et al, and the ATACS Research Group Prospective comparison of unstable angina versus non-Q wave myocardial infarction during antithrombotic therapy. 7 Am Coll Cardiol 1933;22:1338-43.

4 Rehr R, Disciascio G, Vetrovec G, Cowley M. Angiographic morphology of coronary artery stenoses in prolonged rest angina: evidence of intracoronary thrombosis. $\mathscr{f}$ Am Coll Cardiol 1989;14:1429-37.

5 De Wood MA, Stifter WF, Simpson CS, Spores J, Eugster GS, Judge TP, et al. Coronary angiographic findings soon after non-Q wave myocardial infarction. $N$ Engl $f$ Med 1986;315:417-23.

6 Davies MJ, Thomas AC. Plaque fissuring - the cause of acute myocardial infarction, sudden ischaemic death, and crescendo angina. Br Heart $\mathcal{f} 1985 ; 53: 363-73$.

7 Hamm CW, Ravkilde J, Gerhardt W, Jorgensen P, Peheim $\mathrm{E}$, Ljungdahl $\mathrm{L}$, et al. The prognostic value of serum troponin $\mathrm{T}$ in unstable angina. $N$ Engl $f$ Med 1992; ponin $T$ in $146-50$.

8 Theroux P, Latour J-G, Lager-Gauthier C, DeLara J. Fibrinopeptide $A$ and platelet factor levels in unstable angina pectoris. Circulation 1987;75:156-62

9 Kruskal JB, Commerford PJ, Franks JJ, Kirsch RE. Fibrin and fibrinogen-related antigens in patients with stable and unstable coronary artery disease. $N \mathrm{Engl} f \mathcal{M e d}$ 1987;317:1361-5.

10 Fitzgerald DJ, Roy L, Catella F, FitzGerald GA. Platelet activation in unstable coronary disease. $N \mathrm{Engl} \mathrm{f} \mathrm{Med}$ 1986;315:983-9.

11 Maseri A, Pesola A, Marzilli M, Severi S, Parodi O, L'Abbate $A$ et al Coronary vasospasm in angina pectoris. Lancet 1977;i:713-7.

12 Yanagisawa $M$, Kurihara $H$, Kimura $S$, Tomobe $Y$, Kobayashi M, Mitsui Y, et al. A novel potent vasoconstrictor peptide produced by vascular endothelial cells. strictor peptide produced

13 Clarke JG, Benjamin N, Larkin SW, Webb DJ, Davies GJ, Maseri A. Endothelin is a potent long-acting vasoconMaseri A. Endothelin is a potent long-acting vaso

14 Yasuda M, Kohno M, Tahara A, Itagane H, Toda I, Akioka $\mathrm{K}$, et al. Circulating immunoreactive endothelin in ischaemic heart disease. Am Heart $¥$ 1990;119:801-6.

15 Lerman A, Edwards BS, Hallett JW, Heublein DM, Sandberg SM, Burnett JC. Circulating and tissue endothelin immunoreactivity in advanced atherosclerosis. N Engl $\mathcal{Y}$ Med 1991;325:997-1001. 
16 Qiu S, Theroux P, Marcil M, Solymoss BC. Plasma endothelin-1 in stable and unstable angina. Cardiology 1993;82:12-9.

17 Braunwald E. Unstable angina. A classification. Circulation $1989 ; 80: 410-4$.

18 Salminen $K$ Tikkanen I, Saijonmaa O, Nieminen $M$ Fyhrquist F, Frick MH. Modulation of coronary tone in acute myocardial infarction by endothelin. Lancet $1989 ;$;ii: 747 .

19 Miyauchi T, Yanagisawa M, Tomizawa T, Sugishita $Y$, Suzuki N, Fujino $M$, et al. Increased plasma concentrations of endothelin-1 and big endothelin-1 in acute myocardial infarction. Lancet 1989; ii:53-4.

20 Stewart DJ, Kubac G, Costello KB, Cernacek P. Increased plasma endothelin-1 in the early hours after myocardial infarction. $₹ \mathrm{Am}$ Coll Cardiol 1991;18:38-43.

21 Ray SG, McMurray JJ, Morton JJ, Dargie HJ. Circulating endothelin in acute ischaemic syndromes. Br Heart $\mathcal{f}$ 1992;67:383-6.

22 Stewart JT, Nisbet JA, Davies MJ. Plasma endothelin in coronary venous blood from patients with either stable or unstable angina. Br Heart $\mathcal{F} 1991$;66:7-9.

23 Haynes WG, Davenport AP, Webb DJ. Endothelin: progress in pharmacology and physiology. Trends Pharmacol Sci 1993;14:225-8.

24 Yoshizumi $M$, Kurihara $H$, Sugiyama $T$, Takaku $F$, Yanagisawa $M$, Masaki T, Yazaki Y. Hemodynamic shear stress stimulates endothelin production by cultured endothelial cells. Biochem Biophys Res Commun 1989;161:859-64

25 Boulanger C, Luscher TF. Release of endothelin from the porcine aorta: Inhibition by endothelium-derived nitric oxide. 7 Clin Invest 1990;85:587-90.

26 Fyhrquist F, Saijonmaa O, Matsahinne K, Tikkanen I Rosenlof K, Tikkanen T. Raised plasma endothelin-1 concentration following cold pressor test. Biochem Biophys Res Commun 1990;169:217-21.

27 Emori T, Hirata Y, Ohta K, Schichiri M. Secretory mechanism of immunoreactive endothelin in cultured bovine endothelial cells. Biochem Biophys Res Commun 1989; 160:93-100

28 Kurihara $H$, Yoshizumi $M$, Sugiyama $T$, Takaku $F$, Yanagisawa M, Masaki $\mathrm{T}$, et al. Transforming growth factor- $\beta$ stimulates the expression of endothelin mRNA by vascular endothelial cells. Biochem Biophys Res Commun 1989;159:1435-40.

29 Lerman A, Hildebrand FL, Aarhus LL, Burnett JC Endothelin has biological actions at pathophysiological concentrations. Circulation 1991;83:1808-14.

30 Dockrell MEC, Waugh CJ, Haynes WG, Webb DJ, Williams BC. Effect of endothelin-1 on adrenaline stimulated aggregation of human platelets: evidence in support of a biphasic action [abstract]. $f$ Vasc Res 1992; 29:104.

31 Dubin D, Pratt RE, Cooke JP, Dzau VJ. Endothelin, a potent vasoconstrictor, is a vascular muscle smooth mitogen. Fournal of Vascular Medicine and Biology 1989, 1:150-4.

32 Watanabe T, Suzuki N, Shimamoto N, Fujino M, Imada A. Contribution of endogenous endothelin to the extension of myocardial infarct size in rats. Circ Res 1991;69: sion of 